Review Article

\title{
Cell-Derived Microparticles (MPs) and Their Role in Unexplained Recurrent Pregnancy Loss
}

\author{
RUCHA PATIL and SHRIMATI SHETTY \\ National Institute of Immunohaematology (ICMR), $13^{\text {th }}$ Floor, KEM Hospital, Parel, Mumbai 400012 , \\ India
}

(Received on 31 January 2018; Revised on 14 June 2018; Accepted on 18 June 2018)

\begin{abstract}
Circulating cell-derived microparticles (MPs) are sub-micronic phospholipid vesicles derived from cells of different types in response to various biological processes such as cellular activation and apoptosis. They are mainly biomarkers of activation, damage and stress. Apart from the pro-thrombotic attribute, MPs have many other properties and various functions such as being pro-inflammatory, pro-angiogenic or immunomodulatory; and have been found to have a role in vascular dysfunction, cross talk, inflammation, etc. There are many studies which show the association of specific cellderived MPs to different pathological states. MPs act as possible predictors of disease severity and the origin of MPs help us predict the pathological condition; thus MPs represent promising biomarkers of the disease state. There are several methods forthe detection and characterization of MPs; however the most common and gold standard method is flow cytometry. Other methods include enzyme- linked immunosorbent assay and functional assays which measure procoagulant activity and techniques such as electron microscopy, atomic force microscopy, nanoparticle tracking. However, research in the area of MPs faces many problems with regards to the methodology and pre-analytical variables and thus strict guidelines need to be followed for MP estimation. Elevated procoagulant MPs have been detected in patients with pregnancy complications and unexplained recurrent pregnancy loss. MPs may assist in uteroplacental thrombosis leading to pregnancy complications and miscarriages. A preliminary study reports the beneficial use of anticoagulant therapy in such patients with elevated MPs.Present report summarizes different methods used for the detection and analyses of MPs along with their role in pregnancy complications mainly unexplained recurrent pregnancy loss.
\end{abstract}

Keywords: Cell-Derived Microparticles; Thrombosis; Flow Cytometry; Pregnancy Loss; Anticoagulant Therapy

\section{Introduction}

Circulating cell-derived microparticles (MPs), once perceived as simply "innocent debris" and referred as "platelet dust" in the late sixty's (Wolf, 1967; Boulanger et al., 2011) is now emerging as a novel biomarker in a wide range of clinical settings. They are biomarkers reflecting cell activation and cell death; having many attributes such as being procoagulant, pro-inflammatory, etc. and play a major role in intercellular communication. They are emerging as predictive biomarkers for different clinical diseases; as well as circulating biologically active entity (Jy et $a l ., 2010)$. The research on MPs in different areas is growing at a very rapid pace. The methods for detecting and analyzing these MPs are ever evolving

*Author for Correspondence: E-mail: shrimatishetty@yahoo.com with flow cytometry (FC) technique being the gold standard presently.

One of the hypothesized causes of unexplained and/ or recurrent pregnancy loss (RPL) and other pregnancy complications is hypoxia due to uteroplacental thrombosis (Rai, 2003). Nowadays, both genetic thrombophilia and antiphospholipid antibodies are being assessed for the same reason. But even after these investigations, many cases remain idiopathic. There are studies which now report elevated levels of procoagulant MPs in women with pregnancy complications which may contribute to thrombosis in the uteroplacental vasculature leading to placental insufficiency. If this holds true, the next question that arises is whether anticoagulant therapy (ACT) will prove to be beneficial in these cases. 
In this review, we have shed light on what are these cell-derived MPs; the different methods for MP assessment available, their role in unexplained RPL and the clinical implications.

\section{What are Cell- derived Microparticles (MP)?}

Circulating MPs are sub-micronic phospholipid vesicles, 0.1- $1 \mathrm{im}$ in size, derived from cells of different types in response to various biological processes such as cellular activation and apoptosis. MPs harbor a concentrated set of mRNA, proteins, microRNA, etc. and appear to transfer these components to nearby or remote cells (Garcia et al., 2005; Ratajczak et al., 2006; Hunter et al., 2008; Gibbings et al., 2009). Circulating MPs are found in healthy, normal state in a particular range specific to the parent cell from which they are derived; in the absence of disease these MPs originate from aging cells(Zubairovaet al., 2015).

The mechanism of MP formation involves budding of the outer cell membranes which shows cytoskeletal re-organization and the externalization of phosphatidylserine (PS) on the surface, bestowing MPs their procoagulant activity. Lipid rich microdomains have been implicated in endothelial, platelet and monocyte derived MP formation (Burger et al., 2013). The mechanism of formation and size differentiates MPs from exosomes as shown in Fig. 1. Exosomes originate by the inward budding of the membrane which leads to the formation of multivesicular bodies (size: $30-100 \mathrm{~nm}$ ).
Certain populations of MPs have also been shown to express tissue factor (TF) on their surface (Owens and Mackman, 2011; Lacroix and Dignat, 2012). TF expressing MPs are important for thrombin generation and thus play a vital role in blood clotting in vitro (Hrachovinová et al., 2003) as well as thrombus formation in vivo (Aras et al., 2004; Furie and Furie, 2005; Tesselaaret al., 2007; Khorana et al., 2008; Owens and Mackman, 2011; Van Der Meijden et al., 2012) as shown in Fig. 2. PS expressing MPs are thus used as the main marker to identify and quantitate MPs especially in clinical settings associated with thrombosis.

Apart from the pro-thrombotic attribute, MPs have several properties and functions such as being pro-inflammatory, pro-angiogenic or immunomodulatory and have a role in vascular dysfunction, cross talk, inflammation, etc. (Jy et al., 2010). These MPs contribute to pathological states through different mechanisms such as angiogenesis, inflammation and coagulation.

MPs are derived from different cells like endothelial cells, erythrocytes and leukocytes; however the maximum percentage of total MPs is represented by platelets or megakaryocytes (Horstman and Ahn, 1999; Flaumenhaft et al., 2009; Burnier et al., 2009). MPs derived from different cell types possess different functional capabilities due to variations in proteins and lipids derived from the parent cells (Jimenez et al., 2003; Perez-Pujol et al., 2007; Aleman et al., 2011). There are many studies which

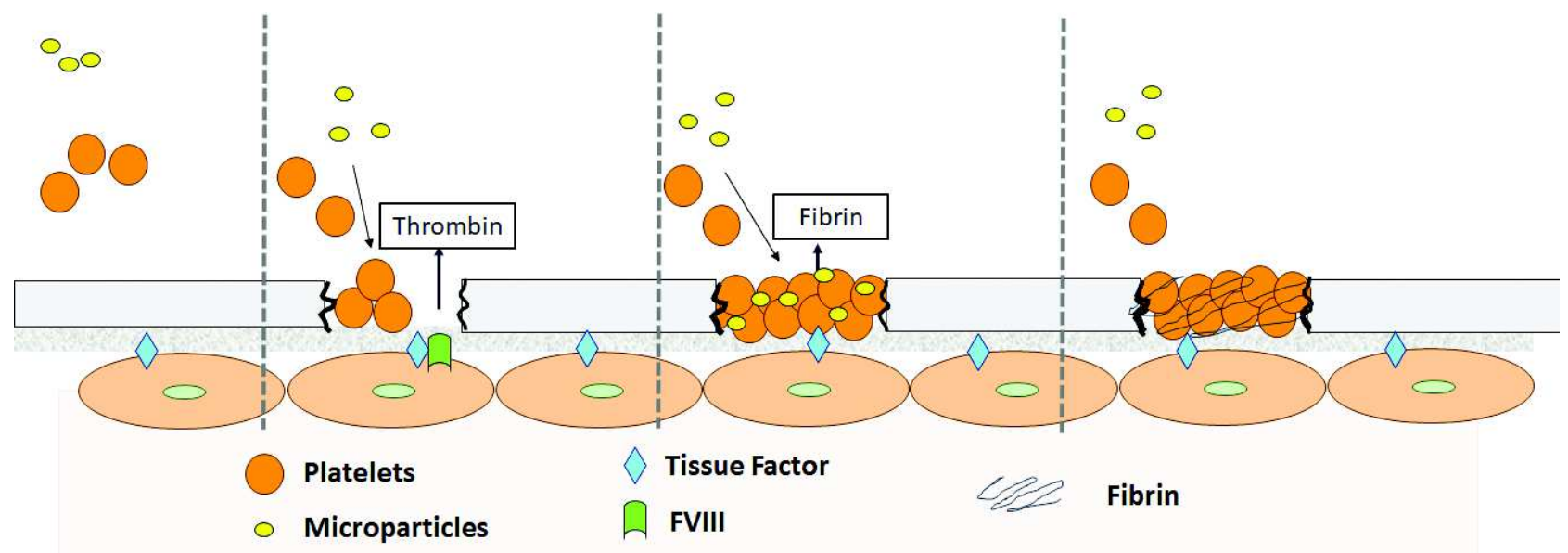

Fig. 1: Mechanism of formation of microparticles and exosomes a) Cell derived microparticles 100-1000 nm: MPs are formed by outward budding of the plasma membrane involves cytoskeletal re-organization and the externalization of phosphatidylserine on the surface, bestowing them their procoagulant activity. b) Exosomes 30- 100 nm: Inward budding of the membrane leads to multivesicular bodies; from which exosomes are released 




Fig. 2: Role of MPs in clot formation: MPs have a role in the formation of clot at the site of blood vessel injury. Once there is an injury to the blood vessel, platelets bind rapidly to the subendothelium and TF expressed on vessel cells activates and triggers the coagulation cascade and binds to FVIII to result in thrombin generation. MPs lead to the amplification of this coagulation cascade; after which fibrin clot is stabilized

have found an association between a particular clinical disorder and elevated MP levels of specific cell type.

However despite these studies on the role of MPs in different diseases, their role in healthy individuals is unclear. MPs are shown to support thrombin generation but whether those in circulation in healthy individuals can provide sufficient activity for the same is unclear (Berckmans et al., 2001). One study showed that MPs have significant causative effects on fibrin polymerization and on the final structure of fibrin clot. MPs accelerate fibrin polymerization and support the formation of lysis resistant hemostatic fibrin clots as well as the thrombi and clots formed in clinical disorder. Therefore, the blood of healthy individuals contains functional MPs at the levels that have a procoagulant potential; they affect the structure and stability of fibrin clots indirectly through acceleration of thrombin generation and through direct physical incorporation into the fibrin network increasing resistance to fibrinolysis (Zubairova et al., 2015).

Research on cell-derived MPs is mainly focused on their potential to act as biomarkers of coagulation, inflammation, endothelial dysfunction, and other pathological processes in different clinical settings to act as predictors. Elevated PS expressing and platelet
MPs (PMPs) is associated with a prothrombotic state, increase in endothelial MPs (EMPs) reflects vascular injury and elevated leucocyte MPs indicates a proinflammatory state. However, as many of these processes overlap, MPs of different type may be elevated or reduced in different pathologies (Burger et al., 2013).

There are many studies which show the association of specific cell-derived MPs to different pathological states. Platelet, leucocyte and endothelial MPs are found elevated in diabetes (Omoto et al., 1999; Omoto et al., 2002; Koga et al., 2005; Feng et al., 2010; Tramontanoet al., 2010) and pulmonary hypertension (Amabile et al., 2008, 2009; Diehl et al., 2011). Elevated MP levels have been found in different thrombotic conditions(Zahra et al., 2011) like atherosclerosis, pulmonary hypertension, heart failure and also end-stage kidney disease (Amabile et al., 2005,2012; Faure et al., 2006); as well as in pregnancy complicated conditions like preeclampsia (PE) (Jadli et al., 2017), intra uterine fetal death, RPL (Patil et al., 2013) etc. Elevated red blood cell MPs have also been found associated with severe dengue(Punyadee et al., 2015).Thus, MPs act as a possible predictor of disease severity; and their origin helps us predict the pathological condition making them promising biomarker of the disease state.

\section{How are these Cell-Derived MPs Detected and Analyzed?}

MPs are shed by all cell populations; however their measurement and detection are restricted to biological fluids. Majority of the studies analyze MP levels in circulating blood; while few have detected MPs in cerebrospinal fluid (Morel et al., 2008), urine (Lytvyn et al., 2017), lung fluids (Roca et al., 2016), sputum (Lacedonia et al., 2016), saliva (Suptawiwat et al., 2017), etc. There are several methods of MP detection and characterization; however the most common method is FC (Flow cytometry). By FC method, one can not only gate the size of MPs but also characterize the cellular origin and quantitate their levels. It also helps in analysing large number of samples in one go. The other common methods of MP detection include enzyme linked immunosorbent assay (ELISA) and functional assays which measure procoagulant activity. Techniques like electron microscopy, atomic force microscopy, nanoparticle tracking have been 
usedfor obtaining detailed phenotypic information but not for bulk sample processing, (Dragovic et al., 2011; van der Pol E et al., 2014). The different methods for MP quantification have been summarized in Fig. 3.

\section{Microparticle Assessment by Flow Cytometry (FC)}

As majority of the cell-derived MPs express PS on their surface, it becomes very easy to detect MPs by flow cytometer. Beads are used to make the gate which includes events of the size $1 \mu \mathrm{m}$ and below. After which these MPs can be tagged with fluorescent conjugated annexin $\mathrm{V}$ along with fluorescent conjugated antibodies for specific cell antigens to enumerate and characterize the cellular origin of the MPs. Flow Cytometer is available in most of the research institutes and many samples can be processed at one go. It is reported that the technique has an intra-assay and inter-assay variability of approximately 2-6\% and 7-12\% respectively (Shet et al., 2003; Simak et al., 2006; Brown et al., 2011).
There had been many debates and concerns regarding the standardization of the $\mathrm{FC}$ technique and its pre-analytical variables. To overcome these problems, a first collaborative workshop to standardize PMP assessment using FC was initiated6 years ago (Lacroix et al., 2010a). The strategy involved the use of forward light scatter (FSC) signal of Megamix beads, Biocytex, which consist of 0.5, 0.9 and $3 \mu \mathrm{m}$ beads so as to gate MPs such a manner that only those events $1 \mu \mathrm{m}$ in size and below are included. Our center has participated in this workshop and standardized the technique on BD FACS Aria. However, it was observed that a better resolution and a more comparable response in SSC oriented instruments was obtained using side scatter signal (SSC) at $90^{\circ} \mathrm{C}$, rather than FSC. The new beads called Megamix SSC beads were then used to make the gates for MPs in such instruments. A new workshop was, thus, initiated by International Society on Thrombosis and Haemostasis (ISTH) Vascular Biology Standardization Subcommittee to evaluate the inter-instrument variability (Cointe et al., 2017). This

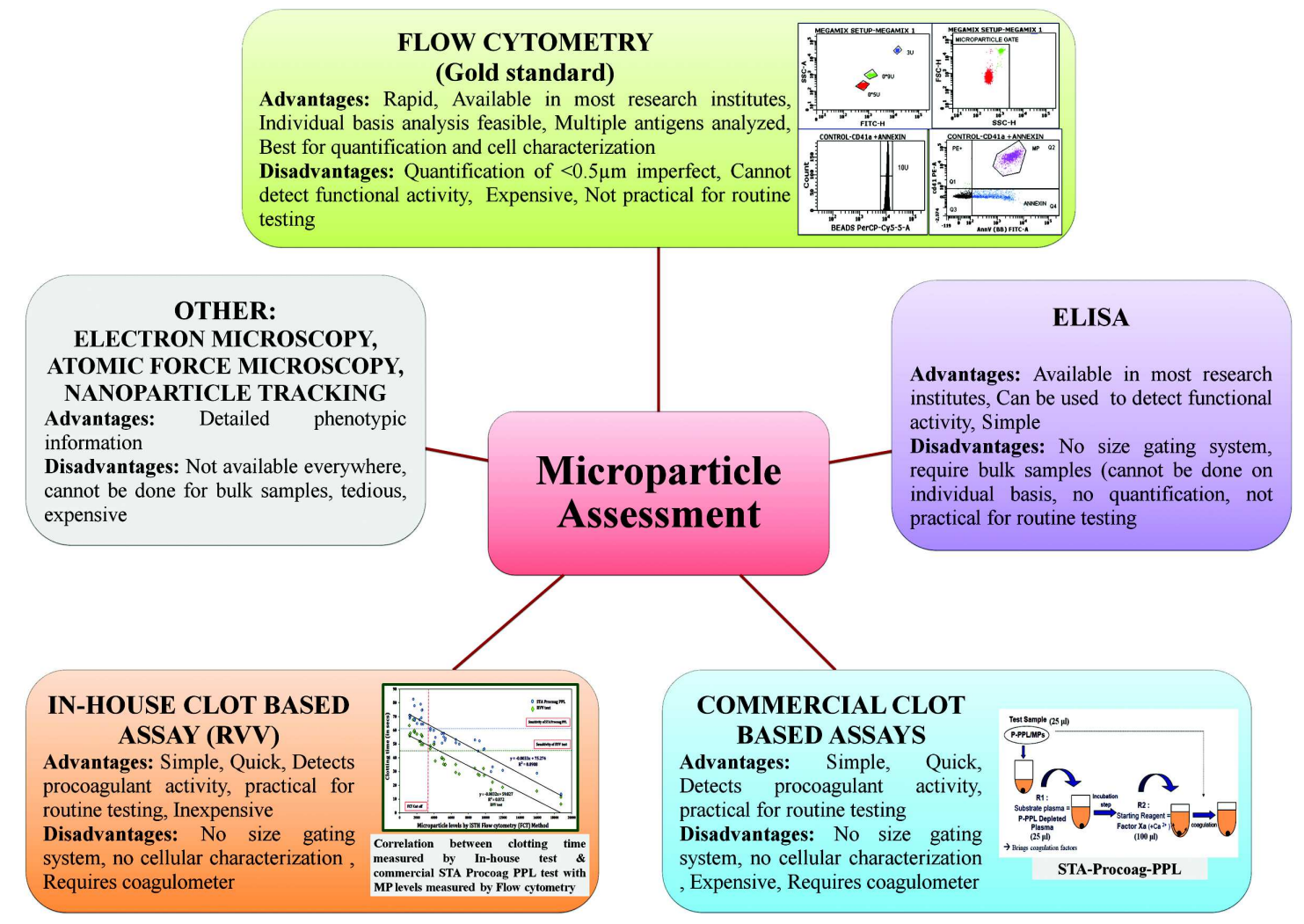

Fig. 3: Pros and Cons of different techniques for microparticle assessment: Flow cytometry is the gold standard method for MP analysis with the major limitation being that it cannot detect functional activity. Thus this method should always be complemented with a functional assay. Other methods used for MP detection are ELISA, clot based assays, and microscopy 
assessment technique for MP analysis using FC is the most reliable method known. One important aspect of this method is the pre-analysis variables and processing which need to be kept uniform (Lacroix et al., 2010b). The disadvantages of the technique are: (a) it works out to be an expensive test and though doable it is not practical for small number of samples to be processed on a daily basis for immediate results and (b) the technique cannot detect functional activity and thus needs to be performed in combination with functional assays.

\section{Enzyme-linked Immune Sorbent Assay (ELISA)}

In this technique, annexin $\mathrm{V}$ is coated in the well which captures PS expressing MPs after which origin of the MPs is detected by using a $2^{\text {nd }}$ antibody specific to surface antigens of cell type. Different captureantibodies and detection- antibodies have been used. This can further be used as a functional assay to check the prothrombinase activity of these MPs by first capturing them and then incubating them with a mixture of factor $\mathrm{Xa}$, factor $\mathrm{Va}$, prothrombin and calcium chloride after which a chromogenic substrate for thrombin is added. The advantages of this technique are that it is simpler, doable in maximum research facilities and allows the detection of procoagulant activity of MPs. The results have been found to correlate with those obtained by FC (Osumi et al., 2001; Hugel et al, 2004; Perez-Casal et al., 2005). However, the major drawback is that as there is no gating system therefore, the size of what is being detected is unknown. Further, there is no insight into the size of MPs being analyzed. Other drawbacks are that the technique requires bulk sample processing rather than individual samples and the functional activity i.e. the prothrombinase activity cannot be assigned completely to MPs; other factors in the plasma may contribute to the same.

\section{Clot Based Assays}

A commercially available clot based assay for MPs i.e. STA Procoag PPL (DiagnosticaStago, France) is an efficient screening technique where in the results correlate well with FC. The method detects procoagulant activity on a semi-automated coagulometer based on the principle that procoagulant MPs will shorten the activated factor X clotting time. It is simple, quick, but expensive. Thus, we aimed to standardize an in-house clot based screening assay for MP detection at our center which would not only be efficient but also inexpensive. In this assay, we measures the clotting time using semi-automated coagulometer after the addition of calcium chloride to MP rich plasma and incubating with Russell viper venom (RVV) and MP poor plasma. RVV activates factor $\mathrm{X}$, thus triggering the coagulation cascade downstream of factor Xa. The MP poor plasma acts like a MP deficient plasma making the test dependent on procoagulant phospholipids on MPs in the samples. The results obtained from this test significantly correlated with the results obtained by FC $\left(\mathrm{R}^{2}=0.87\right.$, $p<0.01)$. Thus, this assay can be utilized routinely for procoagulant MP assessment in various clinical settings. If the clotting time is shortened, FC test can be used to get exact levels and cell origin of MPs (Patil et al., 2016a).

The most important aspect to remember is that irrespective of the technique used for MP assessment, the pre-analytical handling of MPs remains the most important cause of variability in the results. Therefore, the standardized methods need to be strictly adhered to (Vila-Liante et al., 2016; Lacroix et al., 2012).

\section{Potential Role of Cell-derived MPs in Pregnancy Complications}

\section{MPs and Healthy Pregnancy}

Normal pregnancy itself is known to be a hypercoagulable state marked with an increase in the procoagulant activity with elevated levels of factor VII, X, VIII, fibrinogen and vWF; and a decrease in anticoagulants with a major reduction in protein $S$ activity and by acquired activated protein C (APC) resistance. Thus, it is well-known that pregnancy itself as a condition is an acquired risk for thrombosis (Greer, 1999; Heit et al., 2001; Alijotas-Reig et al., 2005). This phenomenon may protect the women from fatal hemorrhage during delivery but may also predispose her to thromboembolism.

In healthy pregnancy, studies suggest that the total platelet, endothelial, leukocyte and TF- bearing MPs as well as the procoagulant activity in healthy non-complicated pregnancy were higher in the $1^{\text {st }}$ trimester as compared to non-pregnant age matched women; these MPs gradually increase during pregnancy with the highest levels observed in the $3^{\text {rd }}$ trimester (Alijotas-Reig et al., 2012; Radu et al., 
2015). Thus, increased MP levels may be one of the contributing factors to the hypercoagulable state seen in healthy pregnancy. There are other reports in which levels of MPs between normal pregnancies and nonpregnant controls do not differ suggesting that MPs are not related to pregnancy. However, MPs associated with fetal-derived human leukocyte antigen were significantly higher in normal pregnant women as compared to non-pregnant controls (Orozco et al., 2009). Lok et al. (2008) observed reduced MPs which subsequently get normalized to the post-partum levels; but placenta-derived MPs were found to have increased.

\section{MPs and Recurrent Pregnancy Loss (RPL)}

Pregnancy loss(PL) is one of the most common complications of pregnancy which affects around $15 \%$ of reproducing couples and recurs in $2-3 \%$ of them. Despite a wide range of investigations, no apparent cause has been found in large number of cases (Coulam, 1991). RPL is defined as 2 or more failed pregnancy, wherein pregnancy is defined as a clinically documented pregnancy by ultrasonography or histopathological test (ASRM, 2013). One hypothesis that exists is that probably a defective maternal hemostatic response may lead to hypoxia and thrombosis of the uteroplacental vasculature and thus assist in subsequent fetal loss. The mechanisms which may be involved in this are impairment of trophoblast invasion, placental microthrombi and villitis (Greer, 1999; Rai, 2003).

Hereditary thrombophilia and antiphospholipid antibodies have found to have a strong association with PL and other pregnancy complications (Voraet al., 2008; Hansda and Roychowdhury, 2012; Ocak et al., 2013; Parand et al., 2013; Chen et al., 2015; Subrt et al., 2013; Ching et al., 2013). For this reason, genetic thrombophilia like protein $\mathrm{C}$, protein $\mathrm{S}$ and antithrombin deficiency, factor $\mathrm{V}$ Leiden mutation; and antiphospholipid antibodies like lupus anticoagulant, anti-cardiolipin antibodies, anti-âa ${ }_{2}^{-}$ glycoprotein-1 antibodies are being tested for in these patients. In a study conducted at our center (Patil et al., 2015a), 587womenwith no apparent etiological causes of RPL and 115 healthy women controls were tested for genetic thrombophilia markers and antiphospholipid antibodies. Among genetic thrombophilia, the risk of PL was highest in women with protein $\mathrm{S}$ deficiency followed byplasminogen activator inhibitor-1 4G/4G polymorphism. Among antiphospholipid antibodies, the risk of PL was the highest in women with anti-cardiolipin antibodies, followed by anti-annexin Vantibodies and lupus anticoagulants. Thus, thrombophilia, both inherited and acquired, is an important contributing factor in unexplained RPL and should be screened.

In the past few years, cell-derived MPs have been found elevated in several prothrombotic conditions and may also be involved in the pathogenesis of RPL. As many cases still remain idiopathic even after being tested for the common thrombophilia markers, MPs seem to be the most plausible candidate for assisting RPL and other pregnancy complications like preeclampsia. Studies show a significant association between circulating EMPs and preeclampsia severity in combination with other markers. Thus, MPs in combination with other markers (serum, etc.) could be used as a good predictor in the $10-14^{\text {th }}$ gestation weeks and to discriminate preeclampsia from other pregnancy complications (VanWijk et al., 2002a, b; González-Quintero et al., 2004; Marques et al., 2012; Jadli et al., 2017).

Majority of the reports have found elevated levels of MP during RPL. Table 1 summarizes reports on cell-derived MPs and recurrent or unexplained PL. Laude et al. (2001) studied 74 women with RPL, 49 being early PL (before $10^{\text {th }}$ gestation week $(\mathrm{GW})$ ) and 25 being late PL (beyond 10th GW) and found that the MP's prothrombotic activity (tested by using prothrombinase assay) is much higher in women with RPL when compared to non-pregnant healthy controls.

Using the technique of FC, Carp et al. (2004) and Pasquier et al. (2013)showed that EMPs increase in women with recurrent miscarriage (RM) and unexplained PL. Kaptan et al. (2008) studied 20 women with RM and showed that PMP levels were significantly higher while the platelet activation marker, P-selectin had marginally increased. In a study done at our center, we found PS expressing TF and EMPs had significantly increased in women with RPL using FC technique and this corresponded with the procoagulant activity of MPs tested by the commercial kit STA Procoag PPL (Patil et al., 2013). Another study (Martinez-Zamora et al., 2016) found significantly higher levels of MPs in women with 
antiphospholipid syndrome (APS) and also in women with unexplained fetal loss when compared to healthy controls; however, there was no statistical difference between these two groups. Contrary to these results, Toth et al. (2008) studied 51 women with RM and did not find significant difference between the test cases and controls with regard to total number of MPs or EMPs or PMPs. Alijotas-Reig et al. (2011) found a significant decrease in EMPs in PL group and RM group; the reason for decrease may be related to its consumption in clotting activation and thus removal from peripheral circulation. The most important point to note in all these studiesis the time at which the sample was assessed for MPs. In the study conducted by Alijotas-Reig et al. (2011) blood samples were collected at the time of diagnosis of the loss and were compared to non-matched healthy pregnant controls. In the above mentioned studies including ours wherein elevated MP levels were observed, the blood samples were collected 4 months after the loss or delivery. This time was chosen as haemostatic changes noted during pregnancy normalize after delivery within 4 to 6 weeks whereas platelet count and protein S takes a little longer to normalize (Hellgren, 2003). In our study, we also found that \% platelet MPs increase and become normal after anticoagulant therapy was initiated in pregnant women with a history of RPL. Thus \% platelet MPs along with PS expressing MPs may be used to assess, diagnose and predict the pregnancy outcome. A decrease in \% platelet MPs may suggest their consumption in fibrin deposit (Patil et al., 2016b).

Thus, these studies show that presence of elevated endothelial, TF and PS expressing MPs suggest a continuous chronic endothelial damage or activation which may eventually get exaggerated at the onset of pregnancy. The data further corroborates that MPs may contribute and assist in uteroplacental thrombosis.

Therefore, in search for an underlying cause of unexplained RPL, focusing on circulating procoagulant MPs seems to be a promising approach. The above mentioned studies clearly show the presence of elevated procoagulant MPs in peripheral circulation in women with early and/or late unexplained/ RPL, thus adding to the new emerging body of evidence that MPs play a significant role in thrombosiscomplicated disorders.

\section{What are the Clinical Implications of Elevated MPs in RPL?}

The next logical question is that if MPs are associated with PL by assisting in developing a prothrombotic state, what clinical approach would be beneficial for these patients? Will ACT which has proven to be beneficial in antiphospholipid syndrome and genetic thrombophilia help prevent thrombosis and thus subsequent PL in patients with elevated levels of procoagulant MPs?

The most debatable topic is whether all women with thrombophilia or unexplained PL need to be anticoagulated during pregnancy. There are contradictory reports with regards to the effect of ACT in pregnancy in women with thrombophilia. Some reports found that the occurrence of PL or pregnancy complications does not decrease with ACT (Rodger et al., 2014; Schleussner et al., 2015) whereas other studies show that ACT such as low dose aspirin, effective and preventive dose of heparin, low or immunosuppressive doses of corticosteroids, etc. are effective in reducing the incidence of adverse pregnancy outcomes in women with thrombophilia or history of idiopathic PL (Rosove et al., 1990; Ghosh Ket al., 2008; Mitiæ et al., 2011; Mutlu et al., 2015).

There are no studies world-wide except a preliminary study done at our center with regards to assessing whether ACT would prove to be beneficial in patients with unexplained/RPL. Bulut et al. (2011) reported that aspirin reduces circulating EMPs and PMPs in patients with cardiovascular disease. In the study done at our center (Patil et al., 2015b), we observed that procoagulant MPs are exaggerated at the onset of pregnancy; in $70 \%$ of these patients exaggerated MP levels decreased significantly as ACT progressed and normalized by the $3^{\text {rd }}$ trimester. These women wherein the MPs normalized had successful live births; possibly as a result of ACT. These patients were on heparin and aspirin. The exact mechanism by which heparin and aspirin reduce the MP levels was not studied. However it is known that heparin has many attributes like being an anticoagulant, antiinflammatory, inhibitory to compliment activation, role in embryonic development, wound healing, metastasis, tissue homeostasis, cell differentiation and proliferation. Aspirin, on the other hand, is an antiplatelet agent that induces platelet aggregation and is a potent vasoconstrictor. Thus, these properties may 


\begin{tabular}{|c|c|c|c|c|}
\hline S.No. & Author & Patients and controls & Results & Method for MP detection \\
\hline 1 & Laude I et al., 2001 & $\begin{array}{l}\text { Patients: } 74 \text { women with unexplained } \\
\left.\text { PL: } 49 \text { early PL (before } 10^{\text {th }} \mathrm{GW}\right) 25 \\
\text { late PL (beyond } 10 \text { th } \mathrm{GW} \text { ) Controls: } \\
50 \text { non-pregnant women }\end{array}$ & $\begin{array}{l}\text { MP's prothrombotic activity higher in women } \\
\text { with RM when compared to non-pregnant } \\
\text { healthy controls }\end{array}$ & Prothrombinase ELISA assay \\
\hline
\end{tabular}

2 Carp H et al., 2004 Patients: 96 women with RPL Controls: 90 parous women

Kaptan et al., 2008 Patients: 20 women with RSA Controls: 20 age matched women

\section{Toth et al., 2008 Patients: 51 women with RSA} Controls: 24 non-pregnant parous women
Patients: 53 women with PL:30 RM16 UFL7 RM + UFLControls: 38 healthy pregnant women 20 healthy non-pregnant women controls

Patients: 124 women with unexplained PLControls: 273 parous women

\section{Patil et al., 2013 Patients: 115 women with RPL} Controls: 20 healthy non-pregnant women
Elevated endothelial MPs were seen in a proportion Flow cytometry using fluorescent of women with RPL (12.5\%) when compared anti-CD51/CD31 antibodies

3-4 months after controls $(2.2 \%)$ suggesting endothelial damage or activation

Platelet MPs were elevated in patients when compared to controls suggesting that PMPs may have a role in pathogenesis of RSA

Total annexin V binding MPs were similar in patients and controls. Elevated levels were observed in 10 patients when compared to 1 in controls. PMP, EMP, thrombin generation and F1+2 did not differ significantly suggesting no direct association between MPs and systemic coagulation activation in RSA patients

Significantly decreased endothelial MP levels were observed in patients with PL when compared to healthy pregnant controls and also in patients with RM when compared to healthy non-pregnant and CD4 $1 \mathrm{MP} / \mu \mathrm{L}$ of plasma

Flow cytometry using platelet $\mathrm{P}$-selectin (CD62P) as a platelet activation marker and $\mathrm{CD} 42 \mathrm{~b}(+)$

Flow cytometry using annexin $\mathrm{V}, \quad$ At least 2 months from and antibodies against CD61, CD63 and CD62P (PMP), as well as CD144 and CD62E (EMP).

Prothrombin fragment $1+2(\mathrm{~F} 1+2)$ and thrombin generation were determined to assess the procoagulant potential of MP

Flow cytometry analyzing total annexin (A5+), endothelial-

At the time clinical diagnosis of miscarriage (CD41+), leukocyte-(CD45+)

Higher endothelial MPs but lower platelet MPs in Flow cytometry using CD51, Time of blood sample collection

At least 2 months from last obstetric event last obstetric event patients than controls suggesting endothelial damage CD144, or CD146 for endothe

CD41 for platelet, CD45 and

CD66b for leukocyte and

neutrophil MPs

Total annexin V, TF and endothelial MPs were found significantly increased in patients when compared to controls. The clot time was also shortened in these samples showing increased procoagulant activity
Flow cytometry (standardized by At least 3 months from participating in the ISTH Vascular last obstetric event Biology SSC workshop) analyzing total annexinV, platelet(CD41a), endothelial (CD146,CD62e), leukocyte (CD45), erythrocyte (CD235a) and tissue factor (CD142) (TF) expressing MPs and STA 
Patil et al., 2015b Patients: 25 women on ACT with history of PL positive for $>1$ thrombophilia but negative for other presumptive aetiological causes. Controls: 25 healthy age matched pregnant women with atleast one live birth and no history of thrombosis

Martinez-Zamora Patients APS group: 50 women et al., 2016 with primary APS with $>3$ consecutive $1^{\text {st }}$ trimester miscarriages. UFL

52 women with with $>3$ consecutive $1^{\text {st }}$ trimester miscarriages of unknown etiology. Control: 52 healthy fertile women with no history of pregnancy loss

Patil et al., 2016b Patients:14 women with unexplained PL on ACT3 women with unexplained PL not on ACTControls: 25 healthy pregnant women
In 15 of the 20 patients who showed exaggerated MPs at the onset of pregnancy, MP levels significantly reduced with ACT as pregnancy progressed and in 14 of them the MP normalized by the $3^{\text {rd }}$ trimester; all of them having successful pregnancy outcome

Cell- derived MPs were found significantly higher in women with APS and with UFL when compared to the control group. However group: there was no statistically significant difference

UFL group

derived MPs

Procoag PPL commercial Clot test

Flow cytometry (standardized by In each trimester and 4 participating in the ISTH Vascular months after pregnancy Biology SSC workshop) analyzing outcome

total annexinV MPs

\%PMPs is decreased at the beginning in women with unexplained PL but after ACT is initiated, the $\%$ increases and normalizes. \%PMPs is highly decreased as pregnancy progresses in women not on therapy with the outcome being PL. \%PMP rather than the PMP levels seem to be a clinically interesting marker along with PS expressing MPs

to assess, diagnose and predict pregnancy outcome.

A decrease in the \%PMPs may suggest their

consumption in fibrin deposits

MP: microparticles; GW: gestation week; PL: pregnancy loss; RM: recurrent miscarriage; RPL: recurrent pregnancy loss; RSA: recurrent spontaneous abortion; PMPs: platelet microparticles; EMP: endothelial microparticles; UFL: unexplained fetal loss; TF: tissue factor; ACT: anticoagulant therapy; APS: Antiphospholipid syndrome; ISTH: International Society of Thrombosis and Haemostasis; SSC: Scientific and standardization committee; STA Procoag PPL kit: Diagnostica Stago, France
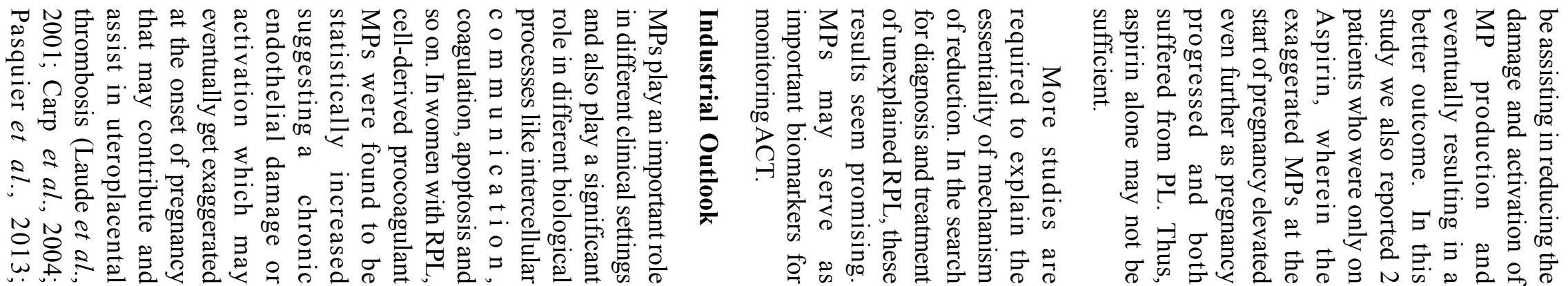
Kaptan et al., 2008; Patil et al., 2013). Similarly MP concentrations are elevated in patients with cardiovascular disease (Baron et al., 2012); MPs are found to be associated with type 2 diabetes which may result in cardiovascular complications (Nomura et al., 2004). Hyper coagulation observed with cancer/ malignancy has been attributed partly to procoagulant MPs (Furie et al., 2005; Kim et al., 2003). Thus, as MPs actively participate in different pathologies, modulation of MP release or clearance may be a novel pharmacological strategy for the management of the disease. In our study, we found exaggerated levels of MPs decreasing as pregnancy progresses in RPL patients on heparin along with aspirin; which resulted in successful pregnancy outcome. However, how heparin and aspirin help in reducing the MP levels is unknown (Patil et al., 2015b). Thus MPs could be potential therapeutic targets and such inhibitors to MPs need to be identified.

Few of the currently identified inhibitors (Baron et al., 2012; Roseblade et al., 2013) include

a) An increase in intracellular calcium plays a key role in MP generation; thus targeting the calcium influx can be one strategy to decrease MP production. However in different studies wherein calcium channel blockers were used, the outcome was positive but the MP levels were still much higher than healthy controls.

b) Targeting calpain is one strategy due to its critical role in release of MPs.

c) Clinical studies have shown the benefit of statins in cardiovascular diseases not only by reducing cholesterol, but possibly due to other modes like endothelial function, vascular inflammation, platelet aggregation, etc. Studies show that statins also reduce MP levels. The level of MPs were found reduced by anti-oxidant treatment reflecting a reduction in platelet activation as well as improvement in endothelial function.

d) Anti-platelet drugs have been used for treatment of hypercoagulability in patients with diabetes mellitus. However, just like calcium channel blockers, the drugs are unable to reduce the MP concentration as compared to that of healthy controls.

MPs being a promising biomarker in different clinical settings have even resulted in the standardization of different tests for their analysis like ELISAs, STA Procoag PPL, and other clot based simple screening assay. Novel diagnostic assays for analyzing different cell-derived MPs which are simple, cost effective as well as sensitive are the need of the hour. Thus, industries can initiate and produce novel methods, reagents and kits for analysis of MPs which can help in different branches of medicine.

Due to its ubiquitous role in multitude of clinical settings, there is a wide scope for industries to target this area; however being a new marker with a lot of unresolved issues, there is a need for more research to resolve the unanswered issues in the area of MPs.

\section{Conclusions}

The cell-derived MPs which were considered as futile and insignificant cellular debris in the earlier days are now emerging as novel important biomarkers of endothelial injury, inflammation and thrombosis. Apart from this, they have been found to play a crucial role in cellular communication, thus impacting numerous pathophysiological processes. The strong association with different disorders makes them very important clinical entities with regard to both diagnosis and treatment. However, research on MPs faces many problems such as methodology and pre-analytical variables and thus strict guidelines need to be adhered to for MP estimation. MPs will have true clinical utility as biomarkers of pathology for different disorders only when the methodology and pre-analytical handling of samples have been further standardized along with validation of the methods. Elevated procoagulant MPs have been detected in patients with pregnancy complication and unexplained RPL. This association of elevated procoagulant MPs in pregnancy complications has caught more attention as pregnancy itself being a hypercoagulable state, the presence of thrombophilia markers and elevated MPs may assist in uteroplacental thrombosis that leads to pregnancy complications and loss. Further, a preliminary study reports the reduction in elevated MP levels in women with PL as ACT progresses, where in these patients had successful live birth; highlighting the beneficial use of ACT in such patients with elevated MPs. Future studies may help explain the mechanism of action of ACT in reducing the MP levels. These findings may then be extended to other thrombosis complicated 
diseases wherein MPs have been found elevated. Clearly, a lot of work needs to be done in this emerging and rising field of research and our understanding of these MPs will definitely expand significantly in future.

\section{References}

Aleman M M, Gardiner C, Harrison P and Wolberg A S (2011) Differential contributions of monocyte- and plateletderived microparticles towards thrombin generation and fibrin formation and stability $J$ Thromb Haemost 92251 2261

Alijotas-Reig J and Ferrer-Raventos J C (2005) Recurrent miscarriage and inherited thrombophilia: diagnostic workout and therapeutic management Med Clin (Barc) 125 626631

Alijotas-Reig J, Palacio-Garcia C, Farran-Codina I, Zarzoso C, Cabero-Roura L and Vilardell-Tarres M (2011) Circulating cell-derived microparticles in women with pregnancy loss Am J Reprod Immunol 66 199-208

Alijotas-Reig J, Palacio-Garcia C, Farran-Codina I, RuizRomance M, Llurba E and Vilardell-Tarres M (2012) Circulating cell-derived microparticles in severe preeclampsia and in fetal growth restriction Am J Reprod Immunol 67 140-151

Amabile N, Guerin AP, Leroyer A, Mallat Z, Nguyen C, Boddaert J, London G M, Tedgui A and Boulanger C M (2005) Circulating endothelial microparticles are associated with vascular dysfunction in patients with end-stage renal failure J Am Soc Nephrol 16 3381-3388

Amabile N, Guerin A P, Tedgui A, Boulanger C M and London G M (2012) Predictive value of circulating endothelial microparticles for cardiovascular mortality in end-stage renal failure: a pilot study Nephrol Dial Transplant $\mathbf{2 7}$ 1873-1880

Amabile N, Heiss C, Chang V, Angeli F S, Damon L, Rame E J, McGlothlin D, Grossman W, De Marco T and Yeghiazarians Y (2009) Increased CD62e+ endothelial microparticle levels predict poor outcome in pulmonary hypertension patients. J Heart Lung Transplant 28 10811086

Amabile N, Heiss C, Real W M, Minasi P, McGlothlin D, Rame E J, Grossman W, De Marco T and Yeghiazarians Y (2008) Circulating endothelial microparticle levels predict hemodynamic severity of pulmonary hypertension $\mathrm{Am} \mathrm{J}$ Respir Crit Care Med 177 1268-1275

Aras O, Shet A, Bach R R, Hysjulien J L, Slungaard A, Hebbel R P, Escolar G, Jilma B and Key N S (2004) Induction of microparticle-associated intravascular tissue factor in human endotoxemia Blood 103 4545-4553

Baron M, Boulanger C M, Staels B and Tailleux A (2012) Cellderived microparticles in atherosclerosis: biomarkers and targets for pharmacological modulation? J Cell Mol Med 16 $1365-1376$

Berckmans R J, Nieuwland R, Böing A N, Romijn F P, Hack C E and Sturk A(2001) Cell-derived microparticles circulate in healthy humans and support low grade thrombin generation Thromb Haemost 85 639-646

Boulanger C M and Dignat-George F (2011) Microparticles: an introduction Arterioscler Thromb Vasc Biol 31 2-3

Brown M D, Feairheller D L, Thakkar S, Veerabhadrappa P and Park J Y (2011) Racial differences in tumor necrosis factor$a ́$-induced endothelial microparticles and interleukin-6 production Vasc Health Risk Manag 7 541-550

Bulut D, Becker V and Mügge A (2011) Acetylsalicylate reduces endothelial and platelet-derived microparticles in patients with coronary artery disease Can J Physiol Pharmacol 89 239-244

Burger D, Schock S, Thompson C S, Montezano A C, Hakim A M and Touyz R M (2013) Microparticles: biomarkers and beyond Clin Sci (Lond) 124 423-441

Burnier L, Fontana P, Kwak B R and Angelillo-Scherrer A(2009) Cell-derived microparticles in haemostasis and vascular medicine Thromb Haemost 101 439-451

Carp H, Dardik R, Lubetsky A, Salomon O, Eskaraev R, Rosenthal E and Inbal A (2004) Prevalence of circulating procoagulant microparticles in women with recurrent miscarriage: a casecontrolled study Hum Reprod 19 191-195

Chen H, Nie S and Lu M(2015) Association between plasminogen activator inhibitor-1 gene polymorphisms and recurrent pregnancy loss: a systematic review and metaanalysis Am J Reprod Immunol 73 292-300

Ching Y M, Arip M, Jegasothy R, Baskaran T P, Yusof A Y, Bakhtiar F and Mustafa N (2013) Antiphospholipid antibodies among women experiencing fetal loss Malays $J$ Pathol 35 147-151

Cointe S, Judicone C, Robert S, Mooberry M J, Poncelet P, Wauben M, Nieuwland R, Key N S, Dignat-George F and Lacroix R (2017) Standardization of microparticle enumeration across different flow cytometry platforms: results of a multicenter collaborative workshop J Thromb Haemost 15 187-193

Coulam C B (1991) Epidemiology of recurrent spontaneous abortion Am J Reprod Immunol 26 23-27

Diehl P, Aleker M, Helbing T, Sossong V, Germann M, Sorichter S, Bode C and Moser M (2011) Increased platelet, 
leukocyte and endothelial microparticles predict enhanced coagulation and vascular inflammation in pulmonary hypertension $J$ Thromb Thrombolysis 31 173-179

Dragovic R A, Gardiner C, Brooks A S, Tannetta D S, Ferguson D J, Hole P, Carr B, Redman C W, Harris A L, Dobson P J, Harrison P and Sargent I L (2011) Sizing and phenotyping of cellular vesicles using nanoparticle tracking analysis Nanomedicine 7 780-788

Faure V, Dou L, Sabatier F, Cerini C, Sampol J, Berland Y, Brunet $\mathrm{P}$ and Dignat-George F (2006) Elevation of circulating endothelial microparticles in patients with chronic renal failure $J$ Thromb Haemostasis 4 566-573

Feng B, Chen Y, Luo Y, Chen M, Li X and Ni Y (2010) Circulating level of microparticles and their correlation with arterial elasticity and endothelium-dependent dilation in patients with type 2 diabetes mellitus Atherosclerosis 208 264269

Flaumenhaft R, Dilks J R, Richardson J, Alden E, Patel-Hett S R, Battinelli E, Klement G L, Sola-Visner M and Italiano J E Jr (2009) Megakaryocyte- derived microparticles: direct visualization and disctinction from platelet-derived microparticles Blood 113 1112-1121

Furie B and Furie B C (2005) Thrombus formation in vivo J Clin Invest 115 3355-62

Garcia B A, Smalley D M, Cho H, Shabanowitz J, Ley K and Hunt D F (2005) The platelet microparticle proteome $J$ Proteome Res 4 1516-1521

Ghosh K, Shetty S, Vora S and Salvi V (2008) Successful pregnancy outcome in women with bad obstetric history and recurrent fetal loss due to thrombophilia: effect of unfractionated heparin and low-molecular weight heparin Clin Appl Thromb Hemost 14 174-179

Gibbings D J, Ciaudo C, Erhardt M and Voinnet O (2009) Multivesicular bodies associate with components of miRNA effector complexes and modulate miRNA activity Nat Cell Biol 11 1143-1149

González-Quintero V H, Smarkusky L P, Jiménez J J, Mauro L M, Jy W, Hortsman L L, O'Sullivan M J and Ahn Y S (2004) Elevated plasma endothelial microparticles: preeclampsia versus gestational hypertension Am JObstet Gynecol 191 1418-1424

Greer I A (1999) Thrombosis in pregnancy: maternal and fetal issues Lancet 353 1258-1265

Hansda J and Roychowdhury J (2012) Study of thrombophilia in recurrent pregnancy loss JObstet Gynaecol India 62 53640

Heit J A, Silverstein M D, Mohr D N, Petterson T M, Lohse C M, O'Fallon W M and Melton L J 3rd (2001) The epidemiology of venous thromboembolism in the community Thromb Haemost 86 452-463

Hellgren M (2003) Hemostasis during normal pregnancy and puerperium Semin Thromb Hemost 29 125-130

Horstman L L and Ahn Y S (1999) Platelet microparticles: a wideangle perspective Crit Rev Oncol Hematol 30 111-142

Hrachovinová I, Cambien B, Hafezi-Moghadam A, Kappelmayer J, Camphausen R T, Widom A, Xia L, Kazazian H H Jr, Schaub R G, McEver R P and Wagner D D (2003) Interaction of P-selectin and PSGL-1 generates microparticles that correct hemostasis in a mouse model of hemophilia A Nat Med 9 1020-1025

Hugel B, Zobairi F and Freyssinet J-M (2004) Measuring circulating cell-derived microparticles $J$ Thromb Haemost 2 1846-1847

Hunter MP, Ismail N, Zhang X, Aguda B D, Lee E J, Yu L, Xiao T, Schafer J, Lee M L, Schmittgen T D, Nana-Sinkam S P, Jarjoura D and Marsh C B (2008) Detection of microRNA expression in human peripheral blood microvesicles $P L O S$ One 3 e3694

Jadli A, Ghosh K, Satoskar P, Damania K, Bansal V and Shetty $S$ (2017) Combination of copeptin, placental growth factor and total annexin $\mathrm{V}$ microparticles for prediction of preeclampsia at 10-14 weeks of gestation Placenta 58 $67-73$

Jimenez J J, Jy W, Mauro L M, Soderland C, Horstman L L and Ahn Y S (2003) Endothelial cells release phenotypically and quantitatively distinct microparticles in activation and apoptosis Thromb Res 109 175-180

Jy W, Horstman L L and Ahn Y S (2010) Microparticle size and its relation to composition, functional activity, and clinical significance Semin Thromb Hemost 36 876-880

Kaptan K, Beyan C, Ifran A and Pekel A (2008) Plateletderived microparticle levels in women with recurrent spontaneous abortion Int J Gynaecol Obstet 102 271-274

Khorana A A, Francis C W, Menzies K E, Wang J G, Hyrien O, Hathcock J, Mackman N and Taubman MB (2008) Plasma tissue factor may be predictive of venous thromboembolism in pancreatic cancer $J$ Thromb Haemost 6 1983-1985

Kim H K, Song K S, Park Y S, Kang Y H, Lee Y J, Lee K R, Kim H K, Ryu K W, Bae J M and Kim S (2003) Elevated levels of circulating platelet microparticles, VEGF, IL-6 and RANTES in patients with gastric cancer: possible role of a metastasis predictor Eur J Cancer 39 184-191

Koga H, Sugiyama S, Kugiyama K, Watanabe K, Fukushima H, Tanaka T, Sakamoto T, Yoshimura M, Jinnouchi H and Ogawa H (2005) Elevated levels of VE-cadherin-positive 
endothelial microparticles in patients with type 2 diabetes mellitus and coronary artery disease $J$ Am Coll Cardiol 45 $1622-1630$

Lacedonia D, Carpagnano G E, Trotta T, Palladino G P, Panaro M A, Zoppo L D, Foschino Barbaro M P and Porro C (2016) Microparticles in sputum of COPD patients: a potential biomarker of the disease? Int J Chron Obstruct Pulmon Dis 11 527-533

Lacroix R, Robert S, Poncelet P and Dignat-George F (2010b) Overcoming limitations of microparticle measurement by flow cytometry Semin Thromb Hemostasis 36 807-818

Lacroix R, Robert S, Poncelet P, Kasthuri R S, Key NS and Dignat-George F (2010a) Standardization of plateletderived microparticle enumeration by flow cytometry with calibrated beads: results of the International Society on Thrombosis and Haemostasis SSC Collaborative workshop J Thromb Haemost 8 2571-2574

Lacroix R and Dignat-George F (2012) Microparticles as a circulating source of procoagulant and fibrinolytic activities in the circulation Thromb Res 129 Suppl 2: S27-29

Lacroix R, Judicone C, Poncelet P, Robert S, Arnaud L, Sampol $J$ and Dignat-George F (2012) Impact of preanalytical parameters on the measurement of circulating microparticles: towards standardization of protocol $J$ Thromb Haemost 10 437-446

Laude I, Rongieres-Bertrand C, Boyer-Neumman C, Wolf M, Mairovitz V, Hugel B, Freyssinet J M, Frydman R, Meyer D and Eschwège V (2001) Circulating procoagulant microparticles in women with unexplained pregnancy loss: a new insight Thromb Haemost 85 18-21

Lok C A, Van Der Post J A, Sargent I L, Hau C M, Sturk A, Boer $\mathrm{K}$ and Nieuwland R (2008) Changes in microparticle numbers and cellular origin during pregnancy and preeclampsia Hypertens Pregnancy 27 344-360

Lytvyn Y, Xiao F, Kennedy C R, Perkins B A, Reich H N, Scholey J W, Cherney D Z and Burger D (2017) Assessment of urinary microparticles in normotensive patients with type 1 diabetes Diabetologia 60 581-584

Marques F K, Campos F M, Filho O A, Carvalho A T, Dusse L $\mathrm{M}$ and Gomes K B (2012) Circulating microparticles in severe preeclampsia Clin Chim Acta 414 253-258

Martínez-Zamora MA, Tàssies D, Creus M, Reverter J C, Puerto B, Monteagudo J, Carmona F and Balasch J (2016) Higher levels of procoagulant microparticles in women with recurrent miscarriage are not associated with antiphospholipid antibodies Hum Reprod 31 46-52

Mitiæ G, Novakov Mikiæ A, Povazan L, Mitreski A, Kopitoviæ $\mathrm{V}$ and Vejnoviæ T (2011) Thromboprophylaxis implementation during pregnancy in women with recurrent foetal losses and thrombophilia Med Pregl 64 471-475

Morel N, Morel O, Petit L, Hugel B, Cochard J F, Freyssinet J M, Sztark F and Dabadie P (2008) Generation of procoagulant microparticles in cerebrospinal fluid and peripheral blood after traumatic brain injury J Trauma 64 698-704

Mutlu I, Mutlu M F, Biri A, Bulut B, Erdem M and Erdem A (2015) Effects of anticoagulant therapy on pregnancy outcomes in patients with thrombophilia and previous poor obstetric history Blood Coagul Fibrinolysis 26267 273

Nomura S, Takahashi N, Inami N, Kajiura T, Yamada K, Nakamori $\mathrm{H}$ and Tsuda N (2004) Probucol and ticlopidine: effect on platelet and monocyte activation markers in hyperlipidemic patients with and without type 2 diabetes Atherosclerosis 174 329-335

Ocak Z, Özlü T and Ozyurt O (2013) Association of recurrent pregnancy loss with chromosomal abnormalities and hereditary thrombophilias Afr Health Sci 13 447-452

Omoto S, Nomura S, Shouzu A, Hayakawa T, Shimizu H, Miyake Y, Yonemoto T, Nishikawa M, Fukuhara S and Inada M (1999) Significance of platelet-derived microparticles and activated platelets in diabetic nephropathy Nephron $\mathbf{8 1}$ 271-277

Omoto S, Nomura S, Shouzu A, Nishikawa M, Fukuhara S and Iwasaka T (2002) Detection of monocyte-derived microparticles in patients with Type II diabetes mellitus Diabetologia 45 550-555

Orozco A F, Jorgez C J, Ramos-Perez W D, Popek E J, Yu X, Kozinetz C A, Bischoff F Z and Lewis D E (2009) Placental release of distinct DNA-associated microparticles into maternal circulation: reflective of gestation time and preeclampsia Placenta 30 891-897

Osumi K, Ozeki Y, Saito S, Nagamura Y, Ito H, Kimura Y, Ogura $\mathrm{H}$ and Nomura S (2001) Development and assessment of enzyme immunoassay for platelet-derived microparticles Thromb. Haemostasis 85 326-330

Owens A P 3rd and Mackman N (2011) Microparticles in hemostasis and thrombosis Circ Res 108 1284-1297

Parand A, Zolghadri J, Nezam M, Afrasiabi A, Haghpanah S and Karimi M (2013) Inherited thrombophilia and recurrent pregnancy loss Iran Red Crescent Med J 15 e13708

Pasquier E, De Saint Martin L, Bohec C, Collet M, Dignat George F and Mottier D (2013) Unexplained pregnancy loss: a marker of basal endothelial dysfunction? Fertil Steril 100 1013-1017. 
Patil R, Ghosh K, Damania K, Bansal V, Satoskar P, Darekar A and Shetty $S$ (2015b) Effect of anticoagulant therapy on cell-derived microparticles and pregnancy outcome in women with pregnancy loss Br J Haematol 171 892-896

Patil R, Ghosh K, Mukherjee S and Shetty S (2016b) Does consumption of platelet-derived microparticles in the fibrin clot explain the decrease in their percentage during pregnancy? Blood Cells Mol Dis 57 115-117

Patil R, Ghosh K and Shetty S (2016a) A simple clot based assay for detection of procoagulant cell-derived microparticles Clin Chem Lab Med 54 799-803

Patil R, Ghosh K, Vora S and Shetty S (2015a) Inherited and acquired thrombophilia in Indian women experiencing unexplained recurrent pregnancy loss Blood Cells Mol Dis 55 200-205

Patil R, Ghosh K, Satoskar P and Shetty S (2013) Elevated procoagulant endothelial and tissue factor expressing microparticles in women with recurrent pregnancy loss PLoS One 8 e 81407

Perez-Casal M, Downey C, Fukudome K, Marx G and Toh CH (2005) Activated protein $\mathrm{C}$ induces the release of microparticle-associated endothelial protein $\mathrm{C}$ receptor Blood 105 1515-1522

Perez-Pujol S, Marker P H and Key N S (2007) Platelet microparticles are heterogeneous and highly dependent on the activation mechanism: studies using a new digital flow cytometer Cytometry A 71 38-45

Practice Committee of the American Society for Reproductive Medicine (2013) Source-American Society for Reproductive Medicine, Birmingham, Alabama. Definitions of infertility and recurrent pregnancy loss: a committee opinion Fertil Steril 9963

Punyadee N, Mairiang D, Thiemmeca S, Komoltri C, Pan-Ngum W, Chomanee N, Charngkaew K, Tangthawornchaikul N, Limpitikul W, Vasanawathana S, Malasit P and Avirutnan P (2015) Microparticles provide a novel biomarker to predict severe clinical outcomes of dengue virus infection J Virol 89 1587-1607

Radu CM, Campello E, Spiezia L, Dhima S, Visentin S, Gavasso S, Woodhams B, Cosmi E and Simioni P (2015) Origin and levels of circulating microparticles in normal pregnancy: A longitudinal observation in healthy women Scand J Clin Lab Invest 75 487-495

Rai R (2003) Is miscarriage a coagulopathy? Curr Opin Obstet Gynecol 15 265-268

Ratajczak J, Wysoczynski M, Hayek F, Janowska-Wieczorek A and Ratajczak M Z (2006) Membrane-derived microvesicles: important and underappreciated mediators of cell-to-cell communication Leukemia 20 1487-1495

Roca E, Lacroix R, Judicone C, Laroumagne S, Robert S, Cointe S, Muller A, Kaspi E ${ }^{7}$, Roll P, Brisson A R, Tantucci C, Astoul P and Dignat-George F (2016) Detection of EpCAM-positive microparticles in pleural fluid: A new approach to mini-invasively identify patients with malignant pleural effusions Oncotarget 73357-3366

Rodger M A, Hague W M, Kingdom J, Kahn S R, Karovitch A, Sermer M, Clement A M, Coat S, Chan W S, Said J, Rey E, Robinson S, Khurana R, Demers C, Kovacs M J, Solymoss S, Hinshaw K, Dwyer J, Smith G, McDonald S, Newstead-Angel J, McLeod A, Khandelwal M, Silver R M, Le Gal G, Greer I A, Keely E, Rosene-Montella $\mathrm{K}$, Walker M and Wells PS; TIPPS Investigators (2014) Antepartum dalteparin versus no antepartum dalteparin for the prevention of pregnancy complications in pregnant women with thrombophilia (TIPPS): a multinational openlabel randomised trial Lancet 384 1673-1683

Roseblade A, Luk F, Rawling T, Ung A, Grau G E and Bebawy M (2013) Cell-derived microparticles: new targets in the therapeutic management of disease $J$ Pharm Pharm Sci 16 238-253

Rosove M H, Tabsh K, Wasserstrum N, Howard P, Hahn B H and Kalunian K C (1990) Heparin therapy for pregnant women with lupus anticoagulant or anticardiolipin antibodies Obstet Gynecol 75 630-634

Schleussner E, Kamin G, Seliger G, Rogenhofer N, Ebner S, Toth B, Schenk M, Henes M, Bohlmann M K, Fischer T, Brosteanu O, Bauersachs R and Petroff D; ETHIG II group (2015) Low-molecular-weight heparin for women with unexplained recurrent pregnancy loss: a multicenter trial with a minimization randomization scheme Ann Intern Med 162 601-609

Shet A S, Aras O, Gupta K, Hass M J, Rausch D J, Saba N, Koopmeiners L, Key N S and Hebbel R P (2003) Sickle blood contains tissue factor-positive microparticles derived from endothelial cells and monocytes Blood 102 26782683

Simak J, Gelderman M P, Yu H, Wright V and Baird A E (2006) Circulating endothelial microparticles in acute ischemic stroke: a link to severity, lesion volume and outcome $J$ Thromb Haemost4 1296-1302

Subrt I, Ulcova-Gallova Z, Cerna M, Hejnalova M, Slovanova J, Bibkova K and Micanova Z (2013) Recurrent pregnancy loss, plasminogen activator inhibitor-1 (-675) 4G/5G polymorphism and antiphospholipid antibodies in Czech women Am J Reprod Immunol 70 54-58

Suptawiwat O, Ruangrung K, Boonarkart C, Puthavathana 
P, Maneechotesuwan K, Charngkaew K, Chomanee N and Auewarakul P (2017) Microparticle and anti-influenza activity in human respiratory secretion PLoS One $\mathbf{1 2}$ e0183717

Tesselaar M E, Romijn F P, Van Der Linden I K, Prins F A, Bertina R M and Osanto S (2007) Microparticle-associated tissue factor activity: a link between cancer and thrombosis? $J$ Thromb Haemost 5 520-527

Toth B, Nieuwland R, Kern M, Rogenhofer N, Berkmans R, Rank A, Lohse P, Friese K and Thaler C J (2008) Systemic changes in haemostatic balance are not associated with increased levels of circulating microparticles in women with recurrent spontaneous abortion Am J Reprod Immunol 59 159-166

Tramontano A F, Lyubarova R, Tsiakos J, Palaia T, Deleon JR and Ragolia L (2010) Circulating endothelial microparticles in diabetes mellitus Mediators Inflamm 2010250476

Van Der Meijden PE, Van Schilfgaarde M, Van Oerle R, Renné T, ten Cate H and Spronk HM (2012) Platelet- and erythrocyte-derived microparticles trigger thrombin generation via factor XIIa $J$ Thromb Haemost 10 13551362

van der Pol E, Coumans F A, Grootemaat A E, Gardiner C, Sargent I L, Harrison P, Sturk A, van Leeuwen T G and Nieuwland R (2014) Particle size distribution of exosomes and microvesicles determined by transmission electron microscopy, flow cytometry, nanoparticle tracking analysis and resistive pulse sensing J Thromb Haemost 12 1182-1192
VanWijk M J, Nieuwland R, Boer K, van der Post J A, VanBavel E and Sturk A (2002a) Microparticle sub populations are increased in preeclampsia: Possible involvement in vascular dysfunction? Am JObstet Gynecol 187 450-456

VanWijk M J, Svedas E, Boer K, Nieuwland R, Vanbavel E and Kublickiene K R (2002b) Isolated microparticles, but not whole plasma, from women with preeclampsia impair endothelium-dependent relaxation in isolated myometrial arteries from healthy pregnant women $\mathrm{Am} \mathrm{J} \mathrm{Obstet}$ Gynecol 187 1686-1693

Vila-Liante V, Sánchez-López V, Martínez-Sales V, Ramón-Nuñez LA, Arellano-Orden E, Cano-RuizA, Rodríguez-Martorell F J, Gao L and Otero-Candelera R (2016) Impact of sample processing on the measurement of circulating microparticles: storage and centrifugation parameters Clin Chem Lab Med 54 1759-1767

Vora S, Shetty S, Salvi V, Satoskar P and Ghosh K (2008) Thrombophilia and unexplained pregnancy loss in Indian patients Natl Med J India 21 116-119

Wolf P (1967) The nature and significance of platelet products in human plasma Br J Haematol 13 269-288

Zahra S, Anderson J A, Stirling D and Ludlam C A (2011) Microparticles, malignancy and thrombosis Br J Haematol 152 688-700

Zubairova L D, Nabiullina R M, Nagaswami C, Zuev Y F, Mustafin I G, Litvinov R I and Weisel J W (2015) Circulating Microparticles Alter Formation, Structure, and Properties of Fibrin Clots Sci Rep 517611. 